\title{
GALNT14 genotype, $\alpha$-fetoprotein and therapeutic side effects predict post-chemotherapy survival in patients with advanced hepatocellular carcinoma
}

\author{
WEY-RAN LIN ${ }^{1,2}$, CHAO-WEI HSU ${ }^{1,2}$, YI-CHENG CHEN ${ }^{1,2}$, MING-LING CHANG ${ }^{1,2}$, \\ KUNG-HAO LIANG ${ }^{1}$, YA-HUI HUANG ${ }^{1,2}$ and CHAU-TING YEH $^{1,2}$ \\ ${ }^{1}$ Liver Research Center, Department of Hepato-Gastroenterology, Linkou Chang Gung Memorial Hospital, \\ ${ }^{2}$ Chang Gung University College of Medicine, Taoyuan 333, Taiwan, R.O.C.
}

Received February 17, 2014; Accepted May 9, 2014

DOI: $10.3892 / \mathrm{mco} .2014 .294$

\begin{abstract}
In addition to targeted agents, chemotherapy is currently considered to be a treatment option for patients with advanced hepatocellular carcinoma (HCC); however, it is associated with severe side effects that may limit its clinical use. UDP- $N$-acetyl- $\alpha$-D-galactosamine:polypep tide $N$-acetyl-galactosaminyltransferase 14 (GALNT14) genotype was previously identified as a prognostic marker for HCC patients receiving 5-fluorouracil, mitoxantrone and cisplatin (FMP) combination chemotherapy. The present study aimed to assess clinical parameters and on-treatment side effects as effective predictors for favorable prognosis. A total of 118 patients with HCC receiving split-dose FMP were retrospectively enrolled. The clinical parameters, side effects and GALNT14 genotype were analyzed. The independent predictors for time-to-progression (TTP) and overall survival (OS) were assessed using Cox proportional hazards models. Following categorization, the Kaplan-Meier method was used to compare survival outcomes. Pretreatment $\alpha$-fetoprotein (AFP) $\leq 2,800 \mathrm{ng} / \mathrm{ml}$ (median level), GALNT14 'TT' genotype,
\end{abstract}

Correspondence to: Professor Chau-Ting Yeh, Liver Research Center, Linkou Chang Gung Memorial Hospital, 5 Fu-Shin Street, Taoyuan 333, Taiwan, R.O.C.

E-mail: chautingy@gmail.com

Abbreviations: AFP, $\alpha$-fetoprotein; ALT, alanine transaminase; AST, aspartate transaminase; BCLC, Barcelona Clinic Liver Cancer; CP, Child-Pugh; ECOG, Eastern Cooperative Oncology Group; FMP, 5-fluorouracil, mitoxantrone and cisplatin; GALNT14, UDP- $N$-acetyl$\alpha$-D-galactosamine:polypeptide $N$-acetylgalactosaminyltransferase 14 ; HBsAg, hepatitis B virus surface antigen; HCC, hepatocellular carcinoma; HCV, hepatitis C virus; OS, overall survival; PVT, portal vein thrombosis; SHARP, Sorafenib HCC Assessment Randomized Protocol; SNP, single-nucleotide polymorphism; TACE, transcatheter arterial chemoembolization; TTP, time-to-progression

Key words: hepatocellular carcinoma, $\alpha$-fetoprotein, single-nucleotide polymorphism, chemotherapy, response on-treatment leukopenia and absence of vomiting were identified as independent predictors of a favorable TTP $(\mathrm{P}=0.001$, $0.035,0.008$ and 0.009 , respectively) and OS ( $\mathrm{P}=0.028$, $0.006,0.027$ and 0.013 , respectively). A total of 59 patients with AFP $\leq 2,800 \mathrm{ng} / \mathrm{ml}$ exhibited longer median TTP and OS (3.11 vs. 1.75 months, $\mathrm{P}<0.001$; and 8.14 vs. 3.79 months, $\mathrm{P}<0.001$, respectively). A total of 30 patients with the GALNT14 'TT' genotype exhibited longer median TTP and OS (3.11 vs. 2.11 months, $\mathrm{P}=0.014$; and 5.75 vs. 3.93 months, $\mathrm{P}=0.001$, respectively). Finally, 9 patients $(9 / 118 ; 7.6 \%)$ with all four favorable factors exhibited the longest median TTP and OS (10.64 vs. 2.07 months, $\mathrm{P}=0.002$; and 25.50 vs. 4.50 months, $\mathrm{P}<0.001$, respectively). In conclusion, the AFP level and the GALNT genotype may be considered as pre-therapeutic predictors of a favorable response. When combined with on-treatment leukopenia and absence of vomiting, a subgroup of patients with excellent outcome may be identified.

\section{Introduction}

Advanced hepatocellular carcinoma (HCC) is a fatal disease without curative measures, with a poor overall survival (OS) of $<6$ months (1). Although the management of advanced HCC has significantly changed over the last few years due to improved patient stratification and introduction of novel therapies, it remains debatable which treatment should be considered as the 'standard therapy' for advanced HCC cases (2). Sorafenib is currently recommended for HCC patients with Barcelona Clinic Liver Cancer (BCLC) stage C $(3,4)$. This is based on two large-scale phase III randomized controlled trials. The Sorafenib HCC Assessment Randomized Protocol (SHARP) trial demonstrated that sorafenib, a tyrosine kinase inhibitor, was able to increase median survival from 7.9 to 10.7 months (5). In the corresponding Asia-Pacific study, the effects of sorafenib in delaying time-to-progression (TTP) and improving OS were further validated (6). However, despite the convincing data, the two studies failed to demonstrate a statistically significant benefit of sorafenib in patients with extrahepatic metastasis. No statistically significant beneficial effect was observed in patients with macroscopic vascular invasion in the Asia-Pacific study. Furthermore, the majority of 
subjects enrolled in those studies belonged to Child-Pugh (CP) class A $(95 \%$ in the SHARP trial and $97 \%$ in the Asia-Pacific study). Further studies demonstrated that patients who received sorafenib with suboptimal liver function (CP class B) exhibited a poorer outcome compared to those with CP class A (7-10). As such, in BCLC stage C HCC patients with CP class B and/or extrahepatic metastasis, sorafenib may not be a priority choice and systemic chemotherapy remains a viable option.

Several combinations of existing chemotherapeutic agents have been used for phase II trials in advanced HCC; however, only a few were able to achieve a response rate of $>20 \%$ (11). Among the regimens, the combination of 5-fluorouracil, mitoxantrone and cisplatin (FMP) consistently achieved a response rate of $>20 \%$ in several studies. However, despite a significant response rate, severe side effects were reported, limiting the clinical use of FMP for advanced HCC $(11,12)$. In order to overcome this difficulty, the identification of a reliable marker, capable of predicting therapeutic responses and, thus, preventing unnecessary side effects, is urgently required. To achieve this goal, a pilot genome-wide association study was conducted and a group of single-nucleotide polymorphism (SNP) markers in patients receiving the standard FMP regimen were identified (13). It was demonstrated that the leading marker, rs9679162, located on the intron of the UDP- $N$-acetyl- $\alpha$-D-galactosamine:polypeptide $N$-acetylgalactosaminyltransferase 14 gene (GALNT14) was capable of predicting the therapeutic response, but not OS, in a previous small-scale validation study (13). To minimize the side effects, we conducted a study using a split-dose FMP protocol, based on the metronomic chemotherapy principle (14). The results revealed that the hematological toxicity was significantly reduced with the split-dose FMP regimen, without a significant alteration of the OS.

Recently, a prospective study demonstrated that an SNP on GALNT14 may predict the therapeutic response, as well as OS, in patients receiving chemotherapy with split-dose FMP (15). However, that study did not investigate the effect of other clinical parameters, including side effects, on the therapeutic response to split-dose FMP. In this study, we analyzed the effect of clinical parameters, GALNT14 genotype and side effects on TTP and OS after the first cycle of split-dose FMP regimen. Besides the GALNT14 genotype, we identified pretreatment $\alpha$-fetoprotein (AFP) levels, on-treatment leukopenia and absence of vomiting as independent prognostic factors. These results highlight that, with careful selection, chemotherapy may be an optimal treatment option for a specific group of patients with advanced HCC in BCLC stage C, with $\mathrm{CP}$ class $\mathrm{B}$, portal vein invasion and/or distant metastasis.

\section{Patients and methods}

Patients. This study was approved by the Institutional Review Board of the Chang Gang Memorial Hospital, Taoyuan, Taiwan. Between January, 2007 and December, 2012, a total of 129 patients were diagnosed with advanced HCC in BCLC stage $\mathrm{C}$, with main portal vein thrombosis (PVT), or distant metastasis, or both. Of these patients, 118 received at least one course of therapy and at least one post-treatment imaging evaluation for outcome assessment. The remaining 11 patients were excluded, due to either failure to complete the first course of chemotherapy, inability to assess the outcome, or refusal to sign an informed consent. The clinical parameters recorded included gender, age, hepatitis B virus surface antigen (HBsAg), anti-hepatitis $\mathrm{C}$ virus antibody (anti-HCV), alcoholism, Eastern Cooperative Oncology Group (ECOG) performance status (16) ascites, CP classification, prior treatment, tumor size, PVT and distant metastasis. Biochemistry and hemogram analysis included bilirubin, alanine transaminase, albumin, creatinine, leukocyte count, neutrophil percentage, hemoglobin, platelet count, prothrombin time and AFP.

HCC was diagnosed by biopsy, aspiration cytology and/or high AFP levels (>400 ng/ml), plus two dynamic imaging studies (dynamic computer tomography and angiography).

Split-dose FMP regimen. The split-dose FMP regimen was modified from the standard regimen as previously described (14). The regimen was as follows: 5-fluorouracil was administered continuously via the intravenous route at a dose of $450 \mathrm{mg} / \mathrm{m}^{2}$ on days $1-5$. Mitoxantrone was administered via intravenous infusion at a dose of $3 \mathrm{mg} / \mathrm{m}^{2}$ on day 1 . Cisplatin was administered as an intravenous infusion at a dose of $40 \mathrm{mg} / \mathrm{m}^{2}$ over $2 \mathrm{~h}$ on day 1 with standard hydration. On the ninth day, the biochemical and hematological data were obtained. If the hepatic, renal and hematological data were satisfactory, 1/4 dose of mitoxantrone and cisplatin was administered on days 9 and 10, respectively. However, if the data indicated severe toxicity, the $1 / 4$ doses were further delayed for one week, until recovery. The treatment was repeated every 4-6 weeks until a maximum of six courses. If grade 3/4 neutropenia and/or leukopenia were observed, granulocyte colony-stimulating factor was administered. Concomitant oral antiviral medication for hepatitis B was allowed. None of the patients received concomitant interferon-based therapy.

GALNT14 genotyping. Genotyping of GALNT14 was performed as previously described (15). Briefly, nuclear DNA was extracted and purified from the peripheral blood prior to treatment. The primers were as follows: forward, 5'-TCACGAGGCCAACATTCTAG-3' and reverse, 5'-TTAGATTCTGCATGGCTCAC-3', designed for PCR and direct sequencing of a 172-bp intronic region of GALNT14 covering rs9679162. The SNP was determined by sequencing data from both directions.

Survival and on-treatment side effects evaluation. At least one lesion was measurable prior to treatment in one dimension in all the evaluated patients. The objective tumor response was assessed by computer tomography every 4-8 weeks after the initiation of chemotherapy. OS was calculated from the date of treatment initiation to the date of death or last follow-up. TTP was calculated from the date of treatment initiation to disease progression. Based on the National Cancer Institute Common Terminology Criteria for adverse events version 3.0 (17), on-treatment side effects were evaluated at day 9 of FMP chemotherapy.

Statistical analysis. All the statistical analyses were performed with SPSS version 18.0 software (SPSS, Inc., Chicago, IL, USA). The data are presented as ratios (\%) for dichotomized variables, as means \pm standard deviation for continuous 
Table I. Baseline characteristics of 118 patients with advanced HCC treated by chemotherapy.

\begin{tabular}{|c|c|c|c|c|}
\hline Characteristics & Total series $(n=118)$ & Cirrhosis $(\mathrm{n}=97)$ & Non-cirrhosis $(\mathrm{n}=21)$ & P-value \\
\hline Age (years) & $58.0 \pm 13.7$ & $58.7 \pm 12.9$ & $54.7 \pm 16.6$ & 0.223 \\
\hline Gender, male, n (\%) & $94(79.7)$ & $75(77.3)$ & $19(90.5)$ & 0.101 \\
\hline $\mathrm{HBV}, \mathrm{n}(\%)$ & $71(60.2)$ & $55(56.7)$ & $16(76.2)$ & 0.080 \\
\hline $\mathrm{HCV}, \mathrm{n}(\%)$ & $42(35.6)$ & $40(41.2)$ & $2(9.5)$ & 0.000 \\
\hline ECOG score $(0 / 1 / 2 / 3)$ & $59 / 33 / 25 / 1$ & $49 / 26 / 21 / 1$ & $10 / 7 / 4 / 0$ & 0.929 \\
\hline Alcoholism, n (\%) & $53(44.9)$ & $43(44.3)$ & $10(47.6)$ & 0.786 \\
\hline Ascites, n (\%) & $60(50.8)$ & $56(57.7)$ & $4(19)$ & 0.001 \\
\hline Child-Pugh class (A/B/C) & 2/115/1 & 2/94/1 & $0 / 21 / 0$ & 0.929 \\
\hline GALNT14 TT genotype, n (\%) & $30(25.4)$ & $26(26.8)$ & $4(19)$ & 0.464 \\
\hline \multicolumn{5}{|l|}{ Prior treatment } \\
\hline Resection, $\mathrm{n}(\%)$ & $7(5.9)$ & $6(6.2)$ & $1(4.8)$ & 0.804 \\
\hline TACE, n $(\%)$ & $49(41.5)$ & $40(41.2)$ & $9(42.9)$ & 0.892 \\
\hline Radiotherapy, n (\%) & $7(5.9)$ & $5(5.2)$ & $2(9.5)$ & 0.447 \\
\hline \multicolumn{5}{|l|}{ Tumor status } \\
\hline Largest tumor size $(\mathrm{cm})$ & $7.8 \pm 4.17$ & $7.42 \pm 4.08$ & $9.56 \pm 4.24$ & 0.033 \\
\hline Portal vein thrombosis, $\mathrm{n}(\%)$ & $81(68.6)$ & $68(70.1)$ & $13(61.9)$ & 0.467 \\
\hline Extrahepatic metastasis, $\mathrm{n}(\%)$ & $65(55.1)$ & $48(49.5)$ & $17(81)$ & 0.004 \\
\hline \multicolumn{5}{|l|}{ Biochemistry and hemogram } \\
\hline Total bilirubin (mg/dl) & $2.11 \pm 3.38$ & $2.08 \pm 2.91$ & $2.23 \pm 5.13$ & 0.854 \\
\hline $\operatorname{ALT}(\mathrm{U} / \mathrm{l})$ & $49.42 \pm 34.11$ & $49.14 \pm 31.79$ & $50.71 \pm 44.16$ & 0.849 \\
\hline Albumin $(\mathrm{g} / \mathrm{dl})$ & $3.33 \pm 0.55$ & $3.28 \pm 0.49$ & $3.55 \pm 0.75$ & 0.129 \\
\hline Creatinine (mg/dl) & $0.78 \pm 0.29$ & $0.79 \pm 0.31$ & $0.73 \pm 0.26$ & 0.414 \\
\hline Leukocyte count $\left(\mathrm{x} 10^{3} / \mathrm{ml}\right)$ & $6.43 \pm 2.94$ & $6.11 \pm 2.89$ & $7.92 \pm 2.73$ & 0.010 \\
\hline Neutrophil percentage (\%) & $68.15 \pm 10.40$ & $67.44 \pm 10.89$ & $71.45 \pm 7.05$ & 0.042 \\
\hline Hemoglobin $(\mathrm{g} / \mathrm{dl})$ & $11.71 \pm 1.93$ & $11.62 \pm 1.85$ & $12.15 \pm 2.27$ & 0.258 \\
\hline Platelet count $\left(\times 10^{3} / \mathrm{ml}\right)$ & $184 \pm 121$ & $168 \pm 94$ & $260 \pm 189$ & 0.039 \\
\hline Prothrombin time (sec) & $13.38 \pm 1.72$ & $13.47 \pm 1.76$ & $12.96 \pm 1.45$ & 0.219 \\
\hline$\alpha$-fetoprotein $(\mathrm{ng} / \mathrm{ml})$ & $2,800\left(2.3-7.5 \times 10^{5}\right)$ & $2,632\left(2.3-3.8 \times 10^{5}\right)$ & $2,955\left(3-7.5 \times 10^{5}\right)$ & 0.167 \\
\hline
\end{tabular}

HCC, hepatocellular carcinoma; HBV, hepatitis B virus; HCV, hepatitis C virus; ECOG, Eastern Cooperative Oncology Group; GALNT14, UDP- $N$-acetyl- $\alpha$-D-galactosamine:polypeptide $N$-acetylgalactosaminyltransferase 14 ; TACE, transcatheter arterial chemoembolization; ALT, alanine transaminase.

variables with normal distribution and as median (range) for continuous variables with a non-normal distribution. For comparisons between groups, the Chi-square or Fisher's exact tests were used for dichotomized data, the two-sample Student's t-test was used for continuous variables with normal distribution and the Mann-Whitney U test was used for continuous variables with a non-normal distribution. The parametric data were dichotomized into two groups, with the medians as cut-offs. Univariate and multivariate Cox proportional hazard models were used to estimate TTP and OS for clinical parameters, on-treatment side effects and genotypic variables. Those variables that were statistically significant in the univariate analysis at the level of $\mathrm{P}<0.05$ were included in the multivariate analysis. Following categorization, the Kaplan-Meier method was used to estimate the survival probability between groups and the log-rank test was used to compare survival outcomes. $\mathrm{P}<0.05$ was considered to indicate a statistically significant difference.

\section{Results}

Basic clinical data of patients with advanced HCC. A total of 118 patients were included. The basic clinical characteristics are listed in Table I. Of the 118 patients, 26 (22.0\%) had an ECOG performance status score of $\geq 2$. CP scoring revealed that 115 patients $(97.5 \%)$ were $\mathrm{CP}$ class $\mathrm{B}$. The etiological analysis demonstrated that 71 patients $(60.2 \%)$ were $\mathrm{HBs} A g$-positive, 42 (35.6\%) were positive for anti-HCV, $8(6.8 \%)$ had hepatitis B and C co-infection and $13(11.0 \%)$ had non-B and non-C etiologies. Notably, alcoholism was observed in $37(52.1 \%)$ of the patients with chronic hepatitis B, 14 (33.3\%) of those with hepatitis C, 4 (50.0\%) of those with hepatitis B and C co-infection and in 6 (46.2\%) of those with non-B and non- $\mathrm{C}$ etiologies, suggesting prevalence of a combination etiology of viral infection and alcoholism (data not shown). The GALNT14 genotype analysis revealed that 30 patients $(25.4 \%)$ had the 'TT' genotype. The tumor size 
Table II. Analysis of factors affecting time-to-progression (TTP) and overall survival (OS) using data from all the patients.

\begin{tabular}{|c|c|c|c|c|c|c|c|c|c|}
\hline \multirow[b]{3}{*}{ Parameters } & \multirow[b]{3}{*}{$\mathrm{n}$} & \multicolumn{4}{|c|}{ TTP } & \multicolumn{4}{|c|}{ OS } \\
\hline & & \multirow{2}{*}{$\begin{array}{c}\begin{array}{c}\text { Univariate } \\
\text { analysis }\end{array} \\
\text { P-value }\end{array}$} & \multicolumn{3}{|c|}{ Multivariate analysis } & \multirow{2}{*}{$\begin{array}{c}\begin{array}{c}\text { Univariate } \\
\text { analysis }\end{array} \\
\text { P-value }\end{array}$} & \multicolumn{3}{|c|}{ Multivariate analysis } \\
\hline & & & HR & $95 \% \mathrm{CI}$ & $\mathrm{P}$-value & & HR & $95 \% \mathrm{CI}$ & $\mathrm{P}$-value \\
\hline Age (years) & & 0.021 & 1.442 & $0.961-2.165$ & 0.077 & 0.209 & & & \\
\hline$\leq 58$ & 60 & & & & & & & & \\
\hline$>58$ & 58 & & & & & & & & \\
\hline Gender & & 0.614 & & & & 0.965 & & & \\
\hline Female & 24 & & & & & & & & \\
\hline Male & 94 & & & & & & & & \\
\hline $\mathrm{HBsAg}$ & & 0.539 & & & & 0.351 & & & \\
\hline Negative & 47 & & & & & & & & \\
\hline Positive & 71 & & & & & & & & \\
\hline Anti-HCV & & 0.786 & & & & 0.094 & & & \\
\hline Negative & 76 & & & & & & & & \\
\hline Positive & 42 & & & & & & & & \\
\hline ECOG score & & 0.001 & 1.449 & $0.899-2.334$ & 0.128 & 0.001 & 1.178 & $0.707-1.960$ & 0.530 \\
\hline 0 & 59 & & & & & & & & \\
\hline $1,2,3$ & 59 & & & & & & & & \\
\hline Alcoholism & & 0.179 & & & & 0.145 & & & \\
\hline No & 65 & & & & & & & & \\
\hline Yes & 53 & & & & & & & & \\
\hline Ascites & & 0.023 & 1.101 & $0.705-1.720$ & 0.672 & 0.004 & 1.314 & $0.819-2.108$ & 0.257 \\
\hline No & 58 & & & & & & & & \\
\hline Yes & 60 & & & & & & & & \\
\hline Cirrhosis & & 0.698 & & & & 0.962 & & & \\
\hline No & 21 & & & & & & & & \\
\hline Yes & 97 & & & & & & & & \\
\hline Child-Pugh class & & 0.950 & & & & 0.827 & & & \\
\hline A & 2 & & & & & & & & \\
\hline $\mathrm{B}, \mathrm{C}$ & 116 & & & & & & & & \\
\hline \multicolumn{10}{|l|}{ GALNT14 TT genotype } \\
\hline No & 88 & & & & & & & & \\
\hline Yes & 30 & 0.016 & 0.582 & $0.352-0.963$ & 0.035 & 0.001 & 0.446 & $0.251-0.790$ & 0.006 \\
\hline \multicolumn{10}{|l|}{ Prior treatment } \\
\hline Resection & & 0.573 & & & & 0.272 & & & \\
\hline No & 111 & & & & & & & & \\
\hline Yes & 7 & & & & & & & & \\
\hline TACE & & 0.265 & & & & 0.133 & & & \\
\hline No & 69 & & & & & & & & \\
\hline Yes & 49 & & & & & & & & \\
\hline Radiotherapy & & 0.116 & & & & 0.463 & & & \\
\hline No & 111 & & & & & & & & \\
\hline Yes & 7 & & & & & & & & \\
\hline \multicolumn{10}{|l|}{ Tumor status } \\
\hline Size $(\mathrm{cm})$ & & 0.030 & 1.014 & $0.966-1.065$ & 0.562 & 0.004 & 1.430 & $0.859-2.380$ & 0.170 \\
\hline$\leq 8$ & 63 & & & & & & & & \\
\hline$>8$ & 55 & & & & & & & & \\
\hline Portal vein thrombosis & & 0.522 & & & & 0.227 & & & \\
\hline No & 37 & & & & & & & & \\
\hline Yes & 81 & & & & & & & & \\
\hline
\end{tabular}


Table II. Continued.

\begin{tabular}{|c|c|c|c|c|c|c|c|c|c|c|}
\hline \multirow[b]{3}{*}{ Parameters } & \multirow[b]{3}{*}{$\mathrm{n}$} & \multicolumn{5}{|c|}{ TTP } & \multicolumn{4}{|c|}{ OS } \\
\hline & & \multirow{2}{*}{$\begin{array}{l}\text { Univariate } \\
\text { analysis } \\
\text { P-value }\end{array}$} & \multicolumn{4}{|c|}{ Multivariate analysis } & \multirow{2}{*}{$\begin{array}{l}\text { Univariate } \\
\text { analysis } \\
\text { P-value }\end{array}$} & \multicolumn{3}{|c|}{ Multivariate analysis } \\
\hline & & & HR & $95 \% \mathrm{CI}$ & $P-v$ & & & $\mathrm{HR}$ & $95 \% \mathrm{CI}$ & P-value \\
\hline Metastasis & & 0.115 & & & & & 0.417 & & & \\
\hline No & 53 & & & & & & & & & \\
\hline Yes & 65 & & & & & & & & & \\
\hline \multicolumn{11}{|l|}{ Biochemistry and hemogram } \\
\hline $\begin{array}{l}\text { Total bilirubin }(\mathrm{mg} / \mathrm{dl}) \\
\leq 1.3\end{array}$ & 63 & 0.039 & 1.203 & 0.783 & 847 & 0.399 & 0.008 & 0.928 & $0.539-1.596$ & 0.787 \\
\hline$>1.3$ & 55 & & & & & & & & & \\
\hline $\operatorname{ALT}(\mathrm{U} / \mathrm{l})$ & & 0.193 & & & & & 0.717 & & & \\
\hline$\leq 39.5$ & 59 & & & & & & & & & \\
\hline$>39.5$ & 59 & & & & & & & & & \\
\hline Albumin (g/dl) & & 0.305 & & & & & 0.004 & 0.791 & $0.474-1.320$ & 0.370 \\
\hline$\leq 3.3$ & 65 & & & & & & & & & \\
\hline$>3.3$ & 53 & & & & & & & & & \\
\hline Creatinine (mg/dl) & & 0.522 & & & & & 0.916 & & & \\
\hline$\leq 0.75$ & 59 & & & & & & & & & \\
\hline$>0.75$ & 59 & & & & & & & & & \\
\hline Leukocyte count $\left(\mathrm{x} 10^{3} / \mathrm{ml}\right)$ & & 0.121 & & & & & 0.028 & 1.074 & $0.633-1.822$ & 0.791 \\
\hline$\leq 5.9$ & 61 & & & & & & & & & \\
\hline$>5.9$ & 57 & & & & & & & & & \\
\hline Neutrophil count (\%) & & 0.018 & 0.997 & 0.633 & 569 & 0.988 & 0.001 & 1.107 & $0.638-1.919$ & 0.718 \\
\hline$\leq 68.95$ & 59 & & & & & & & & & \\
\hline$>68.95$ & 59 & & & & & & & & & \\
\hline Hemoglobin $(\mathrm{g} / \mathrm{dl})$ & & 0.281 & & & & & 0.235 & & & \\
\hline$\leq 11.3$ & 61 & & & & & & & & & \\
\hline$>11.3$ & 57 & & & & & & & & & \\
\hline Platelet count $\left(\mathrm{x} 10^{3} / \mathrm{ml}\right)$ & & 0.052 & & & & & 0.058 & & & \\
\hline$\leq 160$ & 60 & & & & & & & & & \\
\hline$>160$ & 58 & & & & & & & & & \\
\hline Prothrombin time (sec) & & 0.063 & & & & & 0.006 & 1.560 & $0.928-2.624$ & 0.094 \\
\hline$\leq 13$ & 59 & & & & & & & & & \\
\hline$>13$ & 59 & & & & & & & & & \\
\hline $\operatorname{AFP}(\mathrm{ng} / \mathrm{ml})$ & & $<0.001$ & 2.019 & 1.318 & 094 & 0.001 & $<0.001$ & 1.706 & $1.059-2.747$ & 0.028 \\
\hline$\leq 2,800$ & 59 & & & & & & & & & \\
\hline$>2,800$ & 59 & & & & & & & & & \\
\hline
\end{tabular}

HR, hazard ratio; CI, confidence interval; HBsAg, hepatitis B virus surface antigen; HCV, hepatitis C virus; ECOG, Eastern Cooperative Oncology Group; GALNT14, UDP- $N$-acetyl- $\alpha$-D-galactosamine:polypeptide $N$-acetylgalactosaminyltransferase 14 ; TACE, transcatheter arterial chemoembolization; ALT, alanine transaminase; AFP, $\alpha$-fetoprotein.

measured by the greatest diameter was $7.8 \pm 4.17 \mathrm{~cm}$. Distant metastasis was identified in 65 patients $(55.1 \%)$, main PVT in $81(68.6 \%)$ and both distant metastasis and PVT in 38 patients (32.2\%), reflecting an advanced stage of HCC. A total of 63 patients $(53.4 \%)$ had received previous treatment, including surgical resection, transcatheter arterial chemoembolization and radiotherapy, whereas none of the patients had previously received systemic chemotherapy or targeted drugs.

The presence of cirrhosis was determined by either computed tomography or ultrasonography, plus the presence of esophageal varices determined by endoscopy. Of the 118 patients, $97(82.2 \%)$ were cirrhotic. Compared to the non-cirrhotic patients, cirrhotic patients exhibited a higher frequency of $\mathrm{HCV}$ infection (41.2 vs. 9.5\%, $\mathrm{P}<0.001$ ), ascites (57.7 vs. $19.0 \%, \mathrm{P}=0.001$ ), smaller tumor size $(7.42 \pm 4.08$ vs. $9.56 \pm 4.24 \mathrm{~cm}, \mathrm{P}=0.033)$, lower metastasis rate $(49.5$ vs. $81 \%, \mathrm{P}=0.004)$, lower leukocyte count $\left(6.11 \pm 2.89\right.$ vs. $\left.7.92 \pm 2.73 \times 10^{3} / \mathrm{ml}, \mathrm{P}=0.01\right)$, lower percentage of neutrophils $(67.44 \pm 10.89$ vs. $71.45 \pm 7.05 \%, \mathrm{P}=0.042)$ and lower platelet count $\left(168 \pm 94\right.$ vs. $\left.260 \pm 189 \times 10^{3} / \mathrm{ml}, \mathrm{P}=0.039\right)$. 
Table III. Analysis of side effects affecting time-to-progression (TTP) and overall survival (OS).

\begin{tabular}{|c|c|c|c|c|c|c|c|c|c|}
\hline \multirow[b]{3}{*}{ Side effects } & \multirow[b]{3}{*}{$\mathrm{n}$} & \multicolumn{4}{|c|}{ TTP } & \multicolumn{4}{|c|}{ OS } \\
\hline & & \multirow{2}{*}{$\begin{array}{c}\begin{array}{c}\text { Univariate } \\
\text { analysis }\end{array} \\
\text { P-value }\end{array}$} & \multicolumn{3}{|c|}{ Multivariate analysis } & \multirow{2}{*}{$\begin{array}{c}\begin{array}{c}\text { Univariate } \\
\text { analysis }\end{array} \\
\text { P-value }\end{array}$} & \multicolumn{3}{|c|}{ Multivariate analysis } \\
\hline & & & HR & $95 \% \mathrm{CI}$ & P-value & & HR & $95 \% \mathrm{CI}$ & P-value \\
\hline $\begin{array}{l}\text { Leukopenia } \\
\text { No } \\
\text { Yes }\end{array}$ & $\begin{array}{l}53 \\
65\end{array}$ & 0.005 & 0.586 & $0.395-0.868$ & 0.008 & 0.001 & 0.517 & $0.288-0.929$ & 0.027 \\
\hline $\begin{array}{l}\text { Neutropenia } \\
\text { No } \\
\text { Yes }\end{array}$ & $\begin{array}{l}71 \\
47\end{array}$ & 0.066 & & & & 0.017 & 1.003 & $0.545-1.847$ & 0.991 \\
\hline $\begin{array}{l}\text { Anemia } \\
\text { No } \\
\text { Yes }\end{array}$ & $\begin{array}{r}17 \\
101\end{array}$ & 0.617 & & & & 0.501 & & & \\
\hline $\begin{array}{l}\text { Thrombocytopenia } \\
\text { No } \\
\text { Yes }\end{array}$ & $\begin{array}{l}49 \\
69\end{array}$ & 0.233 & & & & 0.258 & & & \\
\hline $\begin{array}{l}\text { Nausea } \\
\text { No } \\
\text { Yes }\end{array}$ & $\begin{array}{l}52 \\
66\end{array}$ & 0.314 & & & & 0.354 & & & \\
\hline $\begin{array}{l}\text { Vomiting } \\
\text { No } \\
\text { Yes }\end{array}$ & $\begin{array}{l}86 \\
32\end{array}$ & 0.005 & 1.771 & $1.157-2.712$ & 0.009 & 0.016 & 1.807 & $1.136-2.876$ & 0.013 \\
\hline $\begin{array}{l}\text { Mucositis } \\
\text { No } \\
\text { Yes }\end{array}$ & $\begin{array}{l}91 \\
27\end{array}$ & 0.686 & & & & 0.665 & & & \\
\hline $\begin{array}{l}\text { Diarrhea } \\
\text { No } \\
\text { Yes }\end{array}$ & $\begin{array}{l}89 \\
29\end{array}$ & 0.924 & & & & 0.527 & & & \\
\hline $\begin{array}{l}\text { Alopecia } \\
\text { No } \\
\text { Yes }\end{array}$ & $\begin{array}{r}115 \\
3\end{array}$ & 0.514 & & & & 0.378 & & & \\
\hline $\begin{array}{l}\text { Hepatoxicity } \\
\text { No } \\
\text { Yes }\end{array}$ & $\begin{array}{l}99 \\
19\end{array}$ & 0.600 & & & & 0.150 & & & \\
\hline $\begin{array}{l}\text { Skin rash } \\
\text { No } \\
\text { Yes }\end{array}$ & $\begin{array}{r}110 \\
8\end{array}$ & 0.035 & 2.334 & $1.125-4.844$ & 0.023 & 0.002 & 3.489 & $1.653-7.362$ & 0.001 \\
\hline $\begin{array}{l}\text { Fatigue } \\
\text { No } \\
\text { Yes }\end{array}$ & $\begin{array}{l}27 \\
91\end{array}$ & 0.100 & & & & 0.068 & & & \\
\hline $\begin{array}{l}\text { Renal insufficiency } \\
\text { No } \\
\text { Yes }\end{array}$ & $\begin{array}{r}111 \\
7\end{array}$ & 0.088 & & & & 0.028 & 2.636 & $1.100-6.316$ & 0.030 \\
\hline $\begin{array}{l}\text { Bleeding } \\
\text { No } \\
\text { Yes }\end{array}$ & $\begin{array}{r}103 \\
15\end{array}$ & 0.961 & & & & 0.386 & & & \\
\hline $\begin{array}{l}\text { Infection } \\
\text { No } \\
\text { Yes }\end{array}$ & $\begin{array}{r}101 \\
17\end{array}$ & 0.440 & & & & 0.355 & & & \\
\hline
\end{tabular}

HR, hazard ratio; CI, confidence interval; TTP, time-to-progression; OS, overall survival. 
Table IV. Multivariate analysis of factors affecting time-to-progression (TTP) and overall survival (OS).

\begin{tabular}{|c|c|c|c|c|c|c|c|}
\hline \multirow[b]{2}{*}{ Factors } & \multirow[b]{2}{*}{$\mathrm{n}$} & \multicolumn{3}{|c|}{ TTP } & \multicolumn{3}{|c|}{ OS } \\
\hline & & HR & $95 \% \mathrm{CI}$ & P-value & HR & $95 \% \mathrm{CI}$ & P-value \\
\hline $\operatorname{AFP}(\mathrm{ng} / \mathrm{ml})$ & & 2.300 & $1.508-3.508$ & $<0.001$ & 2.305 & $1.475-3.602$ & $<0.001$ \\
\hline$\leq 2,800$ & 59 & & & & & & \\
\hline$>2,800$ & 59 & & & & & & \\
\hline GALNT14 TT genotype & & 0.564 & $0.349-0.911$ & 0.019 & 0.464 & $0.268-0.803$ & 0.006 \\
\hline No & 88 & & & & & & \\
\hline Yes & 30 & & & & & & \\
\hline Leukopenia & & 0.577 & $0.388-0.859$ & 0.007 & 0.564 & $0.368-0.864$ & 0.009 \\
\hline No & 53 & & & & & & \\
\hline Yes & 65 & & & & & & \\
\hline Vomiting & & 1.679 & $1.098-2.569$ & 0.017 & 1.772 & $1.118-2.807$ & 0.015 \\
\hline No & 86 & & & & & & \\
\hline Yes & 32 & & & & & & \\
\hline Skin rash & & 1.236 & $0.570-2.681$ & 0.591 & 1.748 & $0.805-3.798$ & 0.158 \\
\hline No & 110 & & & & & & \\
\hline Yes & 8 & & & & & & \\
\hline
\end{tabular}

HR, hazard ratio; CI, confidence interval; AFP, $\alpha$-fetoprotein; GALNT14, UDP- $N$-acetyl- $\alpha$-D-galactosamine:polypeptide $N$-acetylgalactos aminyltransferase 14; TTP, time-to-progression; OS, overall survival.

Favorable prognostic predictors for TTP and OS prior to treatment. The clinical parameters and the GALNT14 genotype accessed prior to treatment were analyzed by univariate analysis, followed by multivariate Cox proportional hazard analysis (Table II). It was observed that age $(\mathrm{P}=0.021)$, ECOG score $(\mathrm{P}=0.001)$, presence of ascites $(\mathrm{P}=0.023)$, GALNT14 genotype $(\mathrm{P}=0.016)$, tumor size $(\mathrm{P}=0.030)$, total bilirubin $(\mathrm{P}=0.039)$, percentage of neutrophils $(\mathrm{P}=0.018)$ and AFP levels $(\mathrm{P}<0.001)$ were associated with TTP. Following adjustment for the confounding factors, the multivariate analysis demonstrated that only GALNT14 'TT' genotype $(\mathrm{P}=0.035)$ and AFP $\leq 2,800 \mathrm{ng} /$ $\mathrm{ml}(\mathrm{P}=0.001)$ were independent predictors of a favorable TTP.

The univariate analysis revealed that several factors were associated with OS, including ECOG score $(\mathrm{P}=0.001)$, presence of ascites $(\mathrm{P}=0.004)$, GALNT14 genotype $(\mathrm{P}=0.001)$, tumor size $(\mathrm{P}=0.004)$, total bilirubin $(\mathrm{P}=0.008)$, albumin $(\mathrm{P}=0.004)$, leukocyte count $(\mathrm{P}=0.028)$, percentage of neutrophils $(\mathrm{P}=0.001)$, prothrombin time $(\mathrm{P}=0.006)$ and AFP levels $(\mathrm{P}<0.001)$. On multivariate analysis, the GALNT14 genotype $(\mathrm{P}=0.006)$ and AFP levels $(\mathrm{P}=0.028)$ were identified as independent predictors of OS.

Association between outcome and on-treatment side effects. The on-treatment side effects in our study were leukopenia $(55.1 \%)$, neutropenia $(39.8 \%)$, anemia $(85.6 \%)$, thrombocytopenia $(58.5 \%)$, nausea $(55.9 \%)$, vomiting $(27.1 \%)$, mucositis (22.9\%), diarrhea (24.6\%), alopecia (2.5\%), hepatoxicity (16.1\%), skin rash $(6.8 \%)$, fatigue $(77.1 \%)$, renal insufficiency $(5.9 \%)$, bleeding (12.7\%) and infection (14.4\%). Univariate followed by multivariate Cox proportional hazard analysis was performed to elucidate the association of on-treatment side effects with TTP and OS (Table III). The favorable factors associated with TTP were found to be leukopenia $(\mathrm{P}=0.008)$, absence of vomiting $(\mathrm{P}=0.009)$ and absence of skin rash $(\mathrm{P}=0.023)$. As regards OS, on-treatment leukopenia $(\mathrm{P}=0.027)$, absence of vomiting $(\mathrm{P}=0.013)$, absence of skin rash $(\mathrm{P}=0.001)$ and absence of renal insufficiency $(\mathrm{P}=0.030)$ were identified as favorable factors.

Identification of a subgroup of HCC patients most suitable for FMP therapy. To identify a subgroup of patients with advanced HCC with better TTP and OS, pretreatment AFP levels, GALNT14 genotype, on-treatment leukopenia, vomiting and skin rash were selected for further multivariate Cox proportional hazard analysis (Table IV). The favorable factors associated with TTP and OS were identified as AFP levels (both $\mathrm{P}<0.001$ ), GALANT14 genotype $(\mathrm{P}=0.019$ and 0.006 , respectively), on-treatment leukopenia $(\mathrm{P}=0.007$ and 0.009 , respectively) and absence of vomiting ( $\mathrm{P}=0.017$ and 0.015 , respectively). Four favorable factors were analyzed using the Kaplan-Meier survival method and the log-rank test was used to compare the TTP and OS (Fig. 1). A total of 59 patients with AFP $\leq 2,800 \mathrm{ng} / \mathrm{ml}$ had longer median TTP and OS (3.11 vs. 1.75 months, $\mathrm{P}<0.001$; and 8.14 vs. 3.79 months, $\mathrm{P}<0.001$, respectively). A total of 30 patients with the GALNT14 'TT' genotype had longer median TTP and OS (3.11 vs. 2.11 months, $\mathrm{P}=0.014$; and 5.75 vs. 3.93 months, $\mathrm{P}=0.001$, respectively). A total of 65 patients with leukopenia had longer median TTP and OS (2.75 vs. 2.00 months, $\mathrm{P}=0.004$; and 6.32 vs. 4.07 months, $\mathrm{P}=0.001$, respectively). A total of 86 patients without vomiting had longer median TTP and OS (2.32 vs. 1.82 months, $\mathrm{P}=0.004$; and 5.71 vs. 3.29 months, $\mathrm{P}=0.014$, respectively). Finally, 9 patients $(9 / 118 ; 7.6 \%)$ with all four favorable factors exhibited the longest median TTP and OS (10.64 vs. 2.07 months, $\mathrm{P}=0.002$; and 25.50 vs. 4.50 months, $\mathrm{P}<0.001$, respectively). 

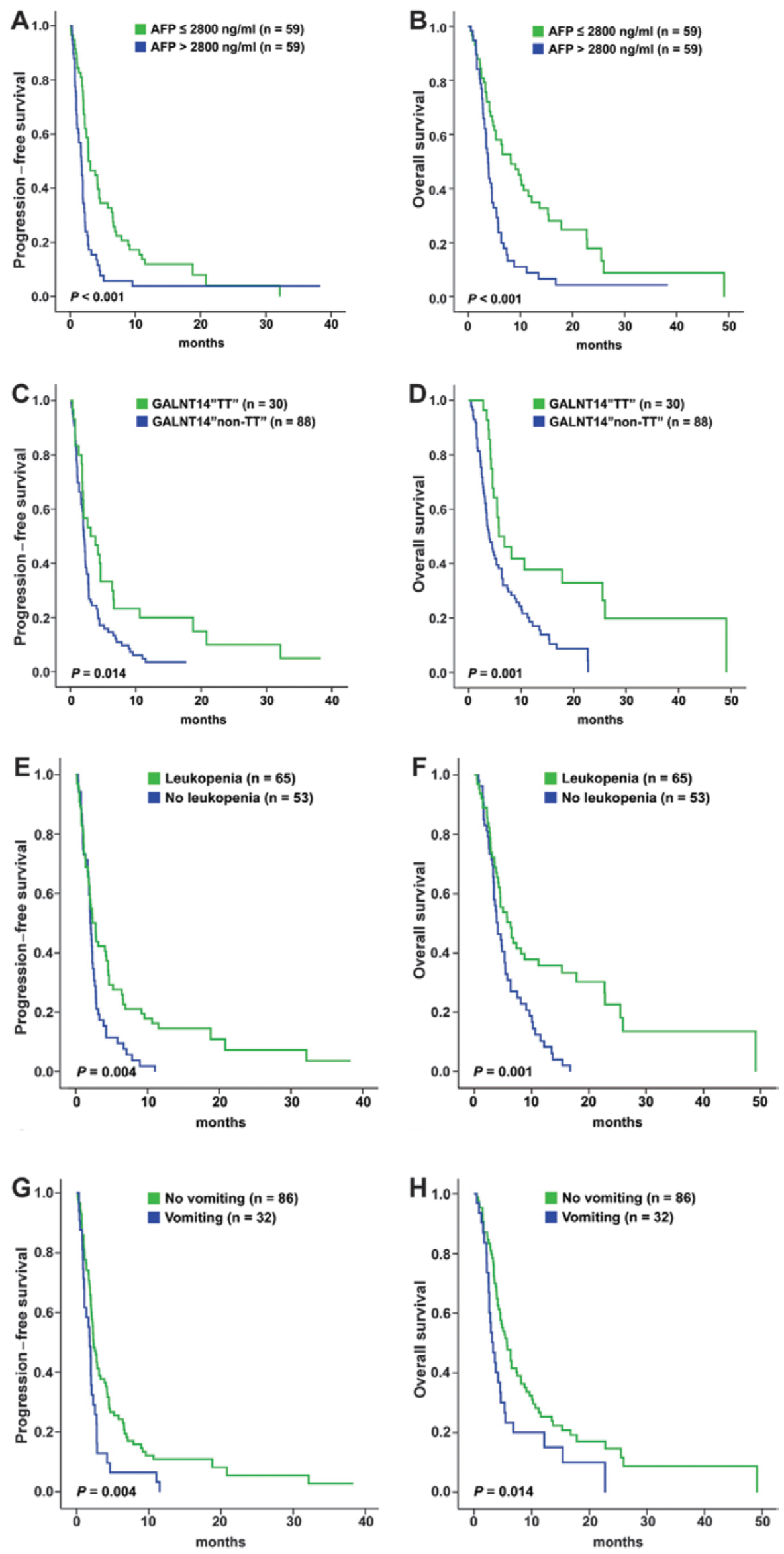

Figure 1. Kaplan-Meier analysis of time-to-progression and overall survival in patients with advanced hepatocellular carcinoma (HCC) receiving split-dose 5-fluorouracil, mitoxantrone and cisplatin (FMP) therapy. (A and B) $\alpha$-fetoprotein (AFP) $\leq 2,800 \mathrm{ng} / \mathrm{ml}$ (green line) vs. AFP $>2,800 \mathrm{ng} / \mathrm{ml}$ (blue line); (C and D) UDP- $N$ acetyl- $\alpha$-D-galactosamine:polypeptide $N$-acetylgalactosaminyltransferase 14 (GALNT14) 'TT' genotype (green line) vs. GALNT14 'non-TT' genotype (blue line); (E and F) on-treatment leukopenia (green line) vs. no leukopenia (blue line); $(\mathrm{G}$ and $\mathrm{H}$ ) absence of vomiting (green line) vs. vomiting (blue line). 

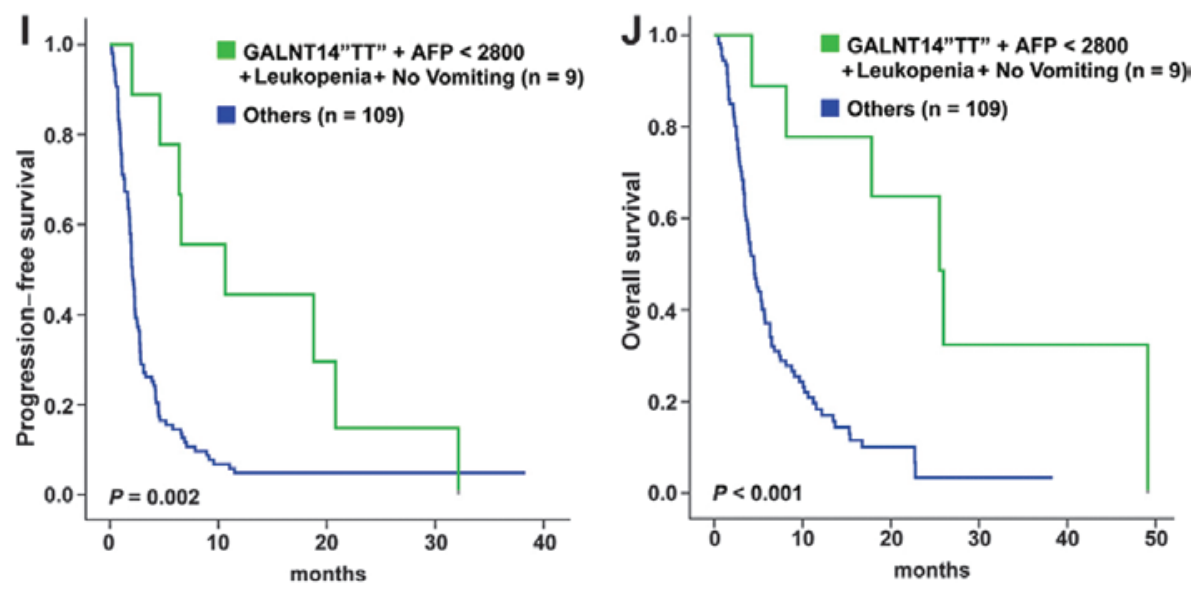

Figure 1. Continued. Kaplan-Meier analysis of time to progression and overall survival in advanced hepatocellular carcinoma (HCC) patients receiving split-dose 5-fluorouracil, mitoxantrone and cisplatin (FMP) therapy. (I and J) selected patients with AFP $\leq 2,800 \mathrm{ng} / \mathrm{ml}$, GALNT14 'TT' genotype, on-treatment leukopenia and absence of vomiting (green line) vs. the remaining patients (blue line).

Since the GALNT14 genotype was identified as a favorable prognostic factor, we further performed Kaplan-Meier survival analysis for GALNT14 'TT' genotype patients with either three of the newly identified factors, or all factors. Among the 30 patients with the GALNT14 'TT' genotype, 13 with AFP levels $\leq 2,800 \mathrm{ng} / \mathrm{ml}$ had longer median TTP and OS (6.51 vs. 1.82 months, $\mathrm{P}=0.049$; and 17.82 vs. 5.39 months, $\mathrm{P}=0.044$, respectively). Of the patients with on-treatment leukopenia, 20 had a longer median OS (17.82 vs. 4.75 months, $\mathrm{P}=0.007)$, but not TTP (4.61 vs. 2.00 months, $\mathrm{P}=0.056$ ). Of the patients without on-treatment vomiting, 24 had a longer median TTP ( 3.86 vs. 1.82 months, $\mathrm{P}=0.041$ ), but not OS (8.14 vs. 4.14 months, $\mathrm{P}=0.070$ ) (data not shown).

A total of 9 patients with the GALNT14 'TT' genotype and three additional favorable factors exhibited the longest median TTP and OS (10.64 vs. 1.96 months, $\mathrm{P}=0.024$; and 25.50 vs. 4.71 months, $\mathrm{P}=0.018$, respectively) (data not shown).

Since pretreatment AFP level as a prognostic factor is easy to obtain in clinical practice, 59 patients with AFP $\leq 2,800 \mathrm{ng} / \mathrm{ml}$ were further analyzed with the Kaplan-Meier survival method using other prognostic factors. A total of 13 patients with the GALNT14 'TT' genotype had longer median TTP and OS (6.57 vs. 2.75 months, $\mathrm{P}=0.017$; and 17.82 vs. 6.50 months, $\mathrm{P}=0.005$, respectively). A total of 33 patients with on-treatment leukopenia had longer median TTP and OS (4.46 vs. 2.50 months, $\mathrm{P}=0.008$; and 17.82 vs. 5.28 months, $\mathrm{P}=0.001$, respectively). A total of 44 patients without on-treatment vomiting had a longer median TTP (4.21 vs. 2.07 months, $\mathrm{P}=0.017$ ), but not OS (9.64 vs. 4.61 months, $\mathrm{P}=0.088$ ). Finally, 9 patients with AFP $\leq 2,800 \mathrm{ng} / \mathrm{ml}$ and three additional favorable factors exhibited the longest median TTP and OS (10.64 vs. 2.79 months, $\mathrm{P}=0.005$; and 25.50 vs. 6.50 months, $\mathrm{P}=0.001$, respectively) (data not shown).

\section{Discussion}

Current guidelines $(3,4)$ recommend sorafenib as the first-line treatment for advanced HCC, based on the results of two phase III randomized controlled trials $(5,6)$. However, not all advanced-stage HCC patients benefit from sorafenib. Those two studies failed to demonstrate statistically significant benefits in patients with extrahepatic spread or poor liver function. Furthermore, a retrospective study on HCC patients treated with soranefib demonstrated a trend towards worse OS from CP class A to B: the median OS was 6.1, 5.4 and 2.7 months in $\mathrm{CP}$ class A, CP class B (score 7) and CP class B patients (score 8 and 9), respectively (8). In the subset of patients with CP class B, the optimal treatment has not been clearly determined. Chemotherapy has been considered as a therapeutic option in patients with advanced HCC; however, the severe side effects and lack of prognostic predictors limit its clinical use. Our group previously demonstrated that the split-dose FMP regimen achieved a similar OS compared to the standard FMP (5.2 vs. 6.0 months, $\mathrm{P}=0.447)$, but was associated with a significantly lower risk of severe neutropenia (5.1 vs. $10.5 \%, \mathrm{P}=0.0005)$ (14). A pilot genome-wide association study and a prospective confirmatory study further verified the predictive value of the GALNT14 genotype $(13,15)$. For advanced HCC patients with CP class B receiving spit-dose FMP therapy, in those with GALNT14 'TT' vs. those with the 'non-TT' genotype, the median TTP was 3.9 vs. 2.1 months, respectively $(\mathrm{P}<0.001)$ and the OS was 6.8 vs. 3.9 months, respectively $(\mathrm{P}<0.001)$.

In this study, we retrospectively investigated 118 patients with advanced $\mathrm{HCC}$ receiving split-dose FMP chemotherapy, 97.5\% of whom were CP class B. Besides the GALNT14 'TT' genotype, we further identified three favorable predictors of outcome, including AFP $\leq 2,800 \mathrm{ng} / \mathrm{ml}$, on-treatment leukopenia and absence of vomiting after the first course of split-dose FMP. By simply using the AFP level, a group with better outcome (median TTP, 3.11 months; OS, 8.14 months) was identified. With the combination of GALNT14 'TT' genotype, an even better median TTP (6.51 months) and OS (17.82 months) may be achieved.Furthermore, on-treatment side effects, including leukopenia and vomiting, may help predict the prognosis. Patients with four favorable factors exhibited the longest median TTP (10.64 months) and OS (25.5 months). Our study provided strong evidence that split-dose FMP chemotherapy may be considered as an effective treatment in patients with advanced $\mathrm{HCC}$ with AFP $\leq 2,800 \mathrm{ng} / \mathrm{ml}$ and/or the GALNT14 'TT' genotype. 
The serum AFP level is a useful marker used for HCC screening and diagnosis worldwide. Although AFP is not elevated in all patients with HCC, a high serum AFP has been associated with advanced tumor stage, including greater tumor size, bilobar involvement, massive or diffuse-type tumor, poorer differentiation and PVT $(18,19)$. The serum AFP level was found to be significantly higher in HCC patients with BCLC stage D compared to stage A and B (18) and was also associated with prognosis. In addition, the AFP level has been considered as an important predictor of postoperative $\mathrm{HCC}$ recurrence and metastasis $(20,21)$. In patients with advanced HCC treated with either sorafenib, transarterial chemoembolization, hepatic artery infusional chemotherapy or concurrent chemoradiotherapy, an early reduction of AFP was found to be a predictor of positive outcome (22-25). Furthermore, the early elevation of AFP was shown to be a predictor of unfavorable outcome in patients with advanced HCC treated with sorafenib (26). In addition, it was demonstrated that a low serum AFP level $(\leq 50 \mathrm{ng} / \mathrm{ml}$ ) was a favorable predictor for patients treated with intravenous 5-fluorouracil and subcutaneous recombinant interferon- $\alpha-2 b$ (27), suggesting a predictive value of AFP in patients receiving chemotherapy. In this study, the patients with BCLC stage C HCC were divided into two groups by the median level of AFP $(2,800 \mathrm{ng} / \mathrm{ml})$. We demonstrated that patients with AFP $\leq 2,800 \mathrm{ng} / \mathrm{ml}$ treated with split-dose FMP regimen had a significantly better prognosis compared to those with AFP $>2,800 \mathrm{ng} / \mathrm{ml}$. This result may be explained in part by the fact that ephrin-A1 expression (an angiogenic factor) in HCC was shown to increase AFP levels and the ability of AFP to elicit the escape of HCC cells from immune surveillance $(28,29)$. However, whether the AFP level is directly associated with chemoresistance of tumor cells remains unclear.

The severe side effects of chemotherapy, such as leukopenia/neutropenia, have been considered as one of the major obstacles for its clinical use. However, it was also demonstrated that there is an association between chemotherapy-induced myelotoxicity and patient outcome in a number of malignancies, including lung, breast, gastric, ovarian and colorectal cancer (30-38). A meta-analysis of 13 trials on various types of cancers $(n=9,528)$ demonstrated a $31 \%$ reduction in the mortality risk for patients with a higher grade of neutropenia or leukopenia, compared to patients with lower-grade or no cytopenia (39). Our findings were consistent with those of previous studies on other malignancies, demonstrating that on-treatment leukopenia is also a prognostic factor in patients with advanced HCC treated with split-dose FMP chemotherapy. It was hypothesized that leukopenia/neutropenia, an indicator of bone marrow suppression caused by a particular dose of a chemotherapeutic agent, may also be a surrogate marker indicating that the same dose is adequate to provide an anticancer effect. Thus, lack of leukopenia or neutropenia may indicate an insufficient or absence of biological effect of chemotherapy.

Of note, the absence of vomiting was also identified as a favorable predictor in this study. Although this information may help predict outcome, the underlying mechanisms have not been elucidated. The presence of vomiting in patients with advanced HCC may be induced by extra-abdominal factors (chemotherapeutic agents, electrolyte abnormalities, central nervous system involvement) or intra-abdominal factors (gastroparesis, ileus, gastric outlet obstruction, bowel obstruction) (40). We hypothesized that on-treatment vomiting indicates poor enteral nutrition in patients with advanced $\mathrm{HCC}$, which may lead to cachexia and compromise the response to antineoplastic therapy.

This study had several notable limitations, including its retrospective nature, limited sampling and restriction of the sample to a Chinese population. Our eligibility criteria confined the treatment regimen to split-dose FMP for interpretative clarity. Therefore, it may not pertain to other chemotherapeutic regimens. It is also unclear whether the leukocyte count measured at day 9 of chemotherapy actually represents the nadir in each patient. However, despite these drawbacks, our study is considered to be suitable for routine clinical practice.

In conclusion, it was recently demonstrated that ideal therapeutic results were not achieved by sorafenib in patients with advanced HCC with CP class B (9). In such patients, we identified a subgroup exhibiting a better clinical outcome when treated with the split-dose FMP chemotherapeutic regimen, simply by assessing the AFP level and/or the GALNT14 genotype. The performance may be further improved by including on-treatment leukopenia and absence of vomiting as predictors. In patients with 4 favorable prognostic factors, a median TTP of $>10$ months and an OS of $>25$ months may be expected.

\section{Acknowledgements}

We would like to thank the staff members of the Liver Research Center for their technical assistance. This study was partly funded by grants from the National Science Council, Taiwan (no. NMRPD1B0052), the Chang Gung University, Taiwan (no. SCRPD1C0071) and the Chang Gung Memorial Hospital, Taiwan (no. CLRPG3C0011).

\section{References}

1. Llovet JM, Brú C and Bruix J: Prognosis of hepatocellular carcinoma: the BCLC staging classification. Semin Liver Dis 19: 329-338, 1999.

2. Villanueva A, Hernandez-Gea V and Llovet JM: Medical therapies for hepatocellular carcinoma: a critical view of the evidence. Nat Rev Gastroenterol Hepatol 10: 34-42, 2013.

3. Bruix J and Sherman M; American Association for the Study of Liver Diseases: Management of hepatocellular carcinoma: an update. Hepatology 53: 1020-1022, 2011.

4. European Association for the Study of the Liver; European Organisation for Research and Treatment of Cancer: EASL-EORTC clinical practice guidelines: management of hepatocellular carcinoma. J Hepatol 56: 908-943, 2012.

5. Llovet JM, Ricci S, Mazzaferro V, et al: Sorafenib in advanced hepatocellular carcinoma. N Engl J Med 359: 378-390, 2008.

6. Cheng AL, Kang YK, Chen Z, et al: Efficacy and safety of sorafenib in patients in the Asia-Pacific region with advanced hepatocellular carcinoma: a phase III randomised, double-blind, placebo-controlled trial. Lancet Oncol 10: 25-34, 2009.

7. Abou-Alfa GK, Amadori D, Santoro A, et al: Safety and efficacy of sorafenib in patients with hepatocellular carcinoma (HCC) and Child-Pugh A versus B cirrhosis. Gastrointest Cancer Res 4: 40-44, 2011.

8. Chiu J, Tang YF, Yao TJ, et al: The use of single-agent sorafenib in the treatment of advanced hepatocellular carcinoma patients with underlying Child-Pugh B liver cirrhosis: a retrospective analysis of efficacy, safety, and survival benefits. Cancer 118: 5293-5301, 2012.

9. Kim HY, Park JW, Joo J, et al: Worse outcome of sorafenib therapy associated with ascites and Child-Pugh score in advanced hepatocellular carcinoma. J Gastroenterol Hepatol 28: 1756-1761, 2013. 
10. Pressiani T, Boni C, Rimassa L, et al: Sorafenib in patients with Child-Pugh class A and B advanced hepatocellular carcinoma: a prospective feasibility analysis. Ann Oncol 24: 406-411, 2013.

11. Zhu AX: Systemic therapy of advanced hepatocellular carcinoma: how hopeful should we be? Oncologist 11: 790-800, 2006.

12. Yang TS, Chang HK, Chen JS, Lin YC, Liau CT and Chang WC: Chemotherapy using 5-fluorouracil, mitoxantrone, and cisplatin for patients with advanced hepatocellular carcinoma: an analysis of 63 cases. J Gastroenterol 39: 362-369, 2004

13. Liang KH, Lin CC and Yeh CT: GALNT14 SNP as a potential predictor of response to combination chemotherapy using 5-FU, mitoxantrone and cisplatin in advanced HCC. Pharmacogenomics 12: 1061-1073, 2011.

14. Yeh CT, Chen HC, Sung CM, et al: Retrospective comparison between a regular and a split-dose protocol of 5-fluorouracil, cisplatin, and mitoxantrone for the treatment of far advanced hepatocellular carcinoma. BMC Cancer 11: 117, 2011.

15. Yeh CT, Liang KH, Lin CC, Chang ML, Hsu CL and Hung CF: A single nucleotide polymorphism on the GALNT14 gene as an effective predictor of response to chemotherapy in advanced hepatocellular carcinoma. Int J Cancer 134: 1214-1224, 2014.

16. Oken MM, Creech RH, Tormey DC, et al: Toxicity and response criteria of the Eastern Cooperative Oncology Group. Am J Clin Oncol 5: 649-655, 1982

17. Trotti A, Colevas AD, Setser A, et al: CTCAE v3.0: development of a comprehensive grading system for the adverse effects of cancer treatment. Semin Radiat Oncol 13: 176-181, 2003.

18. Liu C, Xiao GQ, Yan LN, et al: Value of $\alpha$-fetoprotein in association with clinicopathological features of hepatocellular carcinoma. World J Gastroenterol 19: 1811-1819, 2013.

19. Tangkijvanich P1, Anukulkarnkusol N, Suwangool P, et al: Clinical characteristics and prognosis of hepatocellular carcinoma: analysis based on serum alpha-fetoprotein levels J Clin Gastroenterol 31: 302-308, 2000.

20. Ma WJ, Wang HY and Teng LS: Correlation analysis of preoperative serum alpha-fetoprotein (AFP) level and prognosis of hepatocellular carcinoma (HCC) after hepatectomy. World J Surg Oncol 11: 212, 2013.

21. Chang SK, Hlaing WW, Yu RQ, Lee TW, Ganpathi IS and Madhavan KK: Value of alpha-foetoprotein for screening of recurrence in hepatocellular carcinoma post resection. Singapore Med J 53: 32-35, 2012

22. Lee YK, Kim SU, Kim do Y, et al: Prognostic value of $\alpha$-fetoprotein and des- $\gamma$-carboxy prothrombin responses in patients with hepatocellular carcinoma treated with transarterial chemoembolization. BMC Cancer 13: 5, 2013.

23. Personeni N, Bozzarelli S, Pressiani T, et al: Usefulness of alpha-fetoprotein response in patients treated with sorafenib for advanced hepatocellular carcinoma. J Hepatol 57: 101-107, 2012

24. Memon K, Kulik L, Lewandowski RJ, et al: Alpha-fetoprotein response correlates with EASL response and survival in solitary hepatocellular carcinoma treated with transarterial therapies: a subgroup analysis. J Hepatol 56: 1112-1120, 2012.

25. Lee MH, Kim SU, Kim do Y, et al: Early on-treatment predictions of clinical outcomes using alpha-fetoprotein and des-gamma-carboxy prothrombin responses in patients with advanced hepatocellular carcinoma. J Gastroenterol Hepatol 27: 313-322, 2012
26. Nakazawa T, Hidaka H, Takada J, et al: Early increase in $\alpha$-fetoprotein for predicting unfavorable clinical outcomes in patients with advanced hepatocellular carcinoma treated with sorafenib. Eur J Gastroenterol Hepatol 25: 683-689, 2013.

27. Patt YZ, Yoffe B, Charnsangavej C, et al: Low serum alpha-fetoprotein level in patients with hepatocellular carcinoma as a predictor of response to 5-FU and interferon-alpha- $2 \mathrm{~b}$. Cancer 72: 2574-2582, 1993.

28. Li MS, Ma QL, Chen Q, et al: Alpha-fetoprotein triggers hepatoma cells escaping from immune surveillance through altering the expression of Fas/FasL and tumor necrosis factor related apoptosis-inducing ligand and its receptor of lymphocytes and liver cancer cells. World J Gastroenterol 11: 2564-2569, 2005.

29. Iida H, Honda M, Kawai HF, et al: Ephrin-A1 expression contributes to the malignant characteristics of \{alpha\}-fetoprotein producing hepatocellular carcinoma. Gut 54: 843-851, 2005.

30. Lee CK, Simes RJ, Brown C, et al: Prognostic nomogram to predict progression-free survival in patients with platinum-sensitive recurrent ovarian cancer. Br J Cancer 105: 1144-1150, 2011.

31. Shitara K, Matsuo K, Takahari D, et al: Neutropenia as a prognostic factor in advanced gastric cancer patients undergoing second-line chemotherapy with weekly paclitaxel. Ann Oncol 21: 2403-2409, 2010

32. Shitara K, Matsuo K, Takahari D, et al: Neutropaenia as a prognostic factor in metastatic colorectal cancer patients undergoing chemotherapy with first-line FOLFOX. Eur J Cancer 45: 1757-1763, 2009.

33. Pallis AG, Agelaki S, Kakolyris S, et al: Chemotherapy-induced neutropenia as a prognosticfactor in patients withadvancednon-small cell lung cancer treated with front-line docetaxel-gemcitabine chemotherapy. Lung Cancer 62: 356-363, 2008.

34. Koutras AK, Fountzilas G, Dafni U, et al; Hellenic Cooperative Oncology Group: Myelotoxicity as a prognostic factor in patients with advanced breast cancer treated with chemotherapy: a pooled analysis of two randomised trials conducted by the Hellenic Cooperative Oncology Group. Anticancer Res 28: 2913-2920, 2008.

35. Yamanaka T, Matsumoto S, Teramukai S, Ishiwata R, Nagai Y and Fukushima M: Predictive value of chemotherapy-induced neutropenia for the efficacy of oral fluoropyrimidine S-1 in advanced gastric carcinoma. Br J Cancer 97: 37-42, 2007

36. Di Maio M, Gridelli C, Gallo C, et al: Chemotherapy-induced neutropenia and treatment efficacy in advanced non-small-cell lung cancer: a pooled analysis of three randomised trials. Lancet Oncol 6: 669-677, 2005 .

37. Poikonen P, Saarto T, Lundin J, Joensuu H and Blomqvist C: Leucocyte nadir as a marker for chemotherapy efficacy in node-positive breast cancer treated with adjuvant CMF. Br J Cancer 80: 1763-1766, 1999.

38. Saarto T, Blomqvist C, Rissanen P, Auvinen A and Elomaa I: Haematological toxicity: a marker of adjuvant chemotherapy efficacy in stage II and III breast cancer. Br J Cancer 75: 301-305, 1997.

39. Shitara K, Matsuo K, Oze I, et al: Meta-analysis of neutropenia or leukopenia as a prognostic factor in patients with malignant disease undergoing chemotherapy. Cancer Chemother Pharmacol 68: 301-307, 2011.

40. Lagman RL, Davis MP, LeGrand SB and Walsh D: Common symptoms in advanced cancer. Surg Clin North Am 85: 237-255, 2005. 